\title{
CD73 blockade enhances the local and abscopal effects of radiotherapy in a murine rectal cancer model
}

Hidenori Tsukui

Jichi Ika Daigaku

Hisanaga Horie

Jichi lka Daigaku

Koji Koinuma

Jichi lka Daigaku

Hideyuki Ohzawa

Jichi lka Daigaku

Yasunaru Sakuma

Jichi lka Daigaku

Yoshinori Hosoya

Jichi lka Daigaku

Hironori Yamaguchi

Jichi lka Daigaku

Kotaro Yoshimura

Jichi lka Daigaku

Alan Kawarai Lefor

Jichi lka Daigaku

Naohiro Sata

Jichi lka Daigaku

JOJI KITAYAMA ( $\nabla$ kitayama@jichi.ac.jp )

Jichi Medical University https://orcid.org/0000-0002-5708-7130

Research article

Keywords: abscopal effect, adenosine, CD73, radiation, rectal cancer

Posted Date: March 3rd, 2020

DOI: https://doi.org/10.21203/rs.3.rs-15902/v1 
License: (c) (i) This work is licensed under a Creative Commons Attribution 4.0 International License. Read Full License

Version of Record: A version of this preprint was published at BMC Cancer on May 12th, 2020. See the published version at https://doi.org/10.1186/s12885-020-06893-3. 


\section{Abstract}

\section{Background}

Anti-tumor effects of radiation therapy (RT) largely depend on host immune function. Adenosine with its strong immunosuppressive properties is an important immune checkpoint molecule.

Method

We examined how intra-tumoral adenosine levels modify anti-tumor effects of RT in a murine model using an anti-CD73 antibody which blocks the rate-limiting enzyme to produce extracellular adenosine. We also evaluated CD73 expression in irradiated human rectal cancer tissue.

Results

LuM-1, a highly metastatic murine colon cancer, expresses CD73 with significantly enhanced expression after RT. After subcutaneous (sc) transfer of LuM-1, mice developed macroscopic sc tumors and microscopic pulmonary metastases within 2 weeks. Adenosine levels in the sc tumor were increased after RT. Selective RT (4Gyx3) suppressed the growth of the irradiated sc tumor, but did not affect the growth of lung metastases which were shielded from RT. Intraperitoneal administration of anti-CD73 antibody $(200 \mu \mathrm{g} \times 6)$ alone did not produce antitumor effects. However, when combined with RT in the same protocol, anti-CD73 antibody further delayed the growth of sc tumors and suppressed the development of lung metastases presumably through abscopal effects. Splenocytes derived from RT+ CD73 antibody treated mice showed enhanced IFN-y production and cytotoxicity against LuM-1 compared to controls. Immunohistochemical studies of irradiated human rectal cancer showed that high expression of CD73 in remnant tumor cells and/or stroma is significantly associated with worse outcome.

\section{Conclusion}

These results suggest that adenosine plays an important role in the anti-tumor effects mediated by RT and that CD73/adenosine axis blockade may enhance the anti-tumor effect of RT, and improve the outcomes of patients with locally advanced rectal cancer.

\section{Background}

Neoadjuvant radiation therapy (RT) can down-stage locally advanced rectal cancer (RC) which results in a lower rate of postoperative local recurrences $(1,2)$ and is now considered standard treatment for locally advanced RC worldwide. Recent studies have shown that combined RT and fluorouracil-based chemotherapy results in a further improved locoregional control rate without a significant increase in side effects $(3,4)$. More recently, other radiosensitizers have been used in clinical trials to improve the efficacy and tolerability of RT. 
Although direct cytotoxicity via DNA double-strand breaks or the induction of apoptosis have been considered to be the main mechanisms, a reduction in tumor size is also strongly dependent on host immune responses $(5,6)$. In general, it is believed that RT induces transient immunosuppression. However, multiple reports have suggested that tumor cells which are dead or dying due to RT can present tumor-associated antigens to host immune cells and thereby evoke innate and adaptive immune responses $(7,8)$. This not only increases the cytotoxic effect on tumor cells directly exposed to RT but also causes regression of tumors outside the irradiated field, the so-called "abscopal effect" $(9,10)$.

With the recent remarkable progress in the understanding of immune checkpoint molecules, many studies have been performed to evaluate the efficacy combined RT and immunotherapy. Pre-clinical studies have demonstrated that anti-tumor effects of RT are further enhanced by the concurrent administration of antibodies to CTLA-4 and PD-1 (10-12). Clinical trials have suggested synergistic effects between RT and recently approved antibody preparations against PD-1 and CTLA-4 $(13,14)$. In other clinical studies, however, benefits from combined modality therapy have not been confirmed $(15,16)$. Therefore, the optimal dose or fractionation of RT as well the nature of agents to optimize the response to RT remain to be elucidated.

Adenosine is an important endogenous regulator of innate and the adaptive immune system. Adenosine strongly suppresses immune cells mainly through the A2A receptor and plays a critical role in the maintenance of homeostasis in various tissues $(17,18)$. Adenosine is either released from stressed or injured cells or generated from extracellular adenine nucleotides (ATP, ADP and AMP) by the concerted action of the ectoenzymes ectoapyrase (CD39) and 5'ectonucleotidase (CD73). CD39 catalyzes the hydrolysis of ATP/ADP to AMP and CD73 converts AMP to adenosine, and CD73 mediated conversion is considered to be the rate-limiting enzyme in adenosine production $(19,20)$. ATP is one of the damageassociated molecular patterns (DAMPs) that function as immunostimulatory signals (21). Since adenosine, in contrast, exerts strong immunosuppressive functions, balancing ATP and adenosine is believed to be crucial for the local immune response $(18,22)$.

Malignant cells often express CD73 and high CD73 expression in tumor tissue has been linked to poor clinical outcomes $(23)(24,25)$, suggesting that adenosine produced by the enzymatic activity of CD73 promotes metastases and survival of tumor cells through immunosuppression. In fact, many pre-clinical studies have shown that inhibition of the CD73/adenosine axis can inhibit tumor progression (26-28). Those results suggest that modulation of adenosine levels in the tumor microenvironment can be a novel therapeutic strategy to suppress tumor growth $(29,30)$. In this study, we examined the role of the $\mathrm{CD73/adenosine} \mathrm{axis} \mathrm{on} \mathrm{the} \mathrm{tumor} \mathrm{response} \mathrm{to} \mathrm{local} \mathrm{RT} \mathrm{using} \mathrm{a} \mathrm{murine} \mathrm{model} \mathrm{of} \mathrm{spontaneous} \mathrm{lung}$ metastases and tissue samples from patients with RC.

\section{Methods}

Reagents and mAbs 
Anti-mouse CD73 mAb (clone TY/23) and Rat IgG2a isotype control (clone 2A3) were purchased from BioX-Cell (West Lebanon, NH, USA). Anti-mouse mAbs for flowcytometric analysis were used as described here. FITC-conjugated anti-CD4 (GK1.5), anti-CD8a (53 - 6.7), anti-CD11b (M1/70), and PE-conjugated anti-CD39 (Duha59), anti-IFN-Y (XMG1.2), APC-conjugated anti-CD73 (TY/11.8), anti-CD3 (17A2), and FITC-conjugated anti-CD 45 (30-F11), and mouse recombinant Interleukin-2 (r-IL-2) were purchased from BioLegend (San Diego, CA USA). FcR blocking reagent was obtained from Miltenyi Biotec $\mathrm{GmBH}$ (Bergisch Gladbach, Germany). 7AAD (7-Aminoactinomycin D) and FVS-780 were purchased from Thermo-Fisher Scientific (Waltham, MA USA) and BD Biosciences (Franklin Lakes, NJ USA), respectively.

Cell culture and animal experiments

LuM-1, a highly metastatic sub-clone of murine colon cancer, colon26 (31) was kindly obtained from Dr. Oguri, Aichi Cancer Center, Japan., and maintained in DMEM supplemented with 10\% FCS, $100 \mathrm{U} / \mathrm{mL}$ penicillin and $100 \mu \mathrm{g} / \mathrm{mL}$ streptomycin (Sigma, St. Louis, MO, USA). After achieving $>80 \%$ confluence, cells were removed by treatment with $0.25 \%(\mathrm{w} / \mathrm{v})$ trypsin solution containing $0.04 \%(\mathrm{w} / \mathrm{v})$ EDTA, and then used. The cultured cells were tested by the Mycoplasma Detection Kit (R\&D Systems) in every 3 months and cells with passages 3 to 5 were used for experiments. Female Balb/c mice age 7-8 weeks were purchased from CLEA Japan (Fujinomiya, Japan) and housed in specific pathogen-free (SPF) conditions.

LuM- 1 cells $\left(1 \times 10^{6}\right)$ were subcutaneously $(\mathrm{sc})$ injected in the right flank of 8-9 weeks-old female Balb/c mice. When the primary tumors reached a volume of 100 to $150 \mathrm{~mm}^{3}$ at day 12 , the mice were divided into groups with each group containing $5 \sim 8$ mice to enable the statistical evaluation. Local RT was delivered using MX-160 Labo (mediXtec), as described previously (32). In short, anesthetized mice were held in the decubitus position, and X-ray irradiation was delivered only to the subcutaneous tumor with the remainder of the body of the mouse including the lung covered with a $5 \mathrm{~mm}$ lead plate. We confirmed the effectiveness of shielding by this method. Mice received 3 fractions of 4 Gy every other day (days 12, $14,16)$. For immunotherapy, mice received intraperitoneal (ip) injection of $200 \mu \mathrm{g}$ anti-CD73 mAb or Rat IgG2a isotype control on days 12, 14, 16, 19, 22 and 25 . All of the mice were sacrificed with cervical dislocation on day 28 , and the weight of the sc tumor and number of macroscopic metastatic nodules in lung were evaluated. All the procedures were approved by Animal Care Committee of Jichi Medical University (No 17005-02) and performed according to the Japanese Guidelines for Animal Research.

Flow cytometry

LuM-1 cells were cultured at a density of $1 \times 10^{6}$ cells/10 cm dish and 10 Gy RT given with the MX-160 Labo and incubated for an additional 24 hours. The cells were harvested, incubated with $10 \mu \mathrm{FcR}$ blocking reagent for $10 \mathrm{~min}$ at $4^{\circ} \mathrm{C}$ and incubated with PE-conjugated anti-CD39 (Duha59) and APC antiCD73 mAb for $30 \mathrm{~min}$ at a final concentration of $2.5 \mu \mathrm{g} / \mathrm{mL}$. After washing twice with staining buffer, the cells were incubated with 7-AAD for $15 \mathrm{~min}$ on ice and staining intensity analyzed in 7AAD (-) live cell population using FACS Calibur (BD Bioscience, $\mathrm{NJ}$ ). For in vivo experiments, LuM-1 $\left(1 \times 10^{6}\right)$ cells were sc 
injected in Balb/c mice and treated with 2 fractions of 4 Gy RT as described above. Two days later, tumors were excised and digested using the Tumor Dissociation Kit, mouse (Miltenyi Biotec) with gentleMACs Dissociators (Miltenyi Biotec). After lysis of red blood cells (RBC) with RBC lysis buffer, cells were passed through a 40- $\propto \mathrm{m}$ filter and single cell suspensions stained with APC conjugated anti-CD73 $\mathrm{mAb}$ and FITC conjugated anti-CD45 mAb, and the expression level of CD73 was analyzed in live tumor cell population defined in 7AAD(-) CD45(-) gated area.

T cells producing IFN- $\gamma$ were identified by intracellular staining. Mice bearing sc LuM- 1 tumors received 3 fractions of $4 \mathrm{~Gy}$ local RT and an intraperitoneal injection of $200 \mu \mathrm{g}$ anti-CD73 mAb or Rat IgG2a isotype control every other day (days $12,14,16)$. The mice were sacrificed on day 18 , and splenocytes $\left(1 \times 10^{6}\right)$ were cultured in RPMI-1640 +10\% FCS for 6 hours in the presence of $1 \mu \mathrm{l} / \mathrm{mL}$ brefeldin A for the last 2 hours. The cells were harvested, fixed, permeabilized using the fixation / permeabilization buffer (BD Bioscience) according to the manufacturer's instructions and stained with PE-conjugated IFN- $\gamma$ or isotype control and FITC-conjugated mAbs to CD4 or CD8 as well as FVS780 to exclude dead cells. The ratio of IFN- $\gamma$ positive cells were calculated in $\mathrm{CD} 4(+)$ or $\mathrm{CD} 8(+)$ gated area using LSRFortessa (BD Bioscience).

\section{Cytotoxicity}

Splenocytes $\left(5 \times 10^{6}\right)$ from treated mice (as described above) were cultured with $1 \times 10^{6}$ irradiated (50 Gy) LuM-1 cells in 24-well tissue culture plates in $2 \mathrm{ml} 10 \%$ FCS + PRMI-1640 medium supplemented with $20 \mathrm{ng} / \mathrm{ml}$ mouse rlL-2 for 12 days. Activated splenocytes were incubated with LuM-1 at an E/T ratio of 20:1 for 4 hours and all cells stained with FITC-conjugated Annexin-V, 7-AAD and anti-CD45-APC mAb. The ratio of 7-AAD positive dead cells was calculated in the tumor cell population defined in the FSC/SCC and CD45(-) gated areas.

Quantification of adenosine levels in tumor tissue

Quantitative analysis of adenosine, adenosine monophosphate (AMP) and inosine was performed using an LC-MS system consisting of Nexera X2, LCMS-8060 and a LC/MS/MS Method Package for Primary Metabolite Version 2 (Shimadzu Corp, Kyoto, Japan) as described previously (33). In brief, sc tumors irradiated as described above (4 Gyx2) were resected at 12, 24 and 48 hour after treatment and dissociated using a tumor dissociation kit (Miltenyi Biotec). Chromatographic separation was performed at $40{ }^{\circ} \mathrm{C}$ on a Discovery ${ }^{\circledR}$ HS F5-3 column, $150 \times 2.1 \mathrm{~mm}, 3 \mu \mathrm{m}$, (Sigma-Aldrich) with a flow rate of $0.25 \mathrm{~mL} / \mathrm{min}$. A gradient elution of mobile phase A consisting of $0.1 \%$ of formic acid in water and mobile phase $B$ consisting of $0.1 \%$ of formic acid in acetonitrile. The mobile phase B concentration was programmed as follows: 0\% (0 min) - 0\% (2.0 min) - 25\% (5.0 min) - 35\% (11 min) - 95\% (15 min) $95 \%$ (20 min) - 0\% (20.1 min). Nitrogen gas was used as the nebulizer gas with drying gases at flow rates of 3.0 and $10 \mathrm{~L} / \mathrm{min}$, respectively. Dry air for the heating gas was at $10 \mathrm{~L} / \mathrm{min}$. Collision-induced dissociation (CID) was conducted by argon gas (purity, >99.9995\%). Interface, heat block, and desolvationline temperatures were set at 300,400 , and $250^{\circ} \mathrm{C}$, respectively. Multiple reaction monitoring (MRM) transitions for adenosine, AMP, and inosine were $\mathrm{m} / \mathrm{z} 268.1>136.05, \mathrm{~m} / \mathrm{z} 384.0>136.05 \mathrm{and} \mathrm{m} / \mathrm{z}$ 
$269.1>137.05$, respectively, in positive ion mode. MRM transition for 2-MES was $\mathrm{m} / \mathrm{z} 194.0>80.15$ in negative ion mode. The polarity switching time of the instrument was $5 \mathrm{~ms}$ (10 ms/cycle).

Immunohistochemistry of patient samples

Between 2008 and 2015, 64 patients with locally advanced RC received neoadjuvant chemoradiotherapy (CRT) in the Department of Surgery, Division of Gastroenterological General and Transplant Surgery, Jichi Medical University Hospital. Patients were treated with long-course RT (a dose of 50.4 Gy in 25 fractions) using 4-field box techniques. Some patients received concurrent chemotherapy with oral UFT or S1. Radical resections were performed at 8-10 weeks after the end of CRT. The excised tumors were immediately fixed in $10 \%$ buffered formalin, and consecutive formalin-fixed paraffin-embedded $4-\mu \mathrm{m}$ sections prepared for immunohistochemical evaluation.

After treatment with xylene and ethanol and washing with phosphate-buffered saline (PBS), tumor specimens were subjected to heat-induced antigen retrieval in citrate buffer (Muto Pure Chemicals Co., Ltd, Tokyo, Japan) followed by endogenous peroxidase blocking by Peroxidase-Blocking solution (DAKO, Santa Clara, CA). The tissues were washed with PBS and incubated with $5 \%$ bovine serum albumin for 30 minutes to block nonspecific antibody binding. The slides were then incubated overnight at $4{ }^{\circ} \mathrm{C}$ with monoclonal antibodies against CD73 (D7F9A, Rabbit IgG, Cell Signaling Technology) at a dilution of 1:200 in humid chambers overnight at $4^{\circ} \mathrm{C}$. After three 5-min washes with PBS, sections were incubated with anti-rabbit secondary antibody conjugated with peroxidase for $30 \mathrm{~min}$ at room temperature. After washing, the enzyme substrate 3,30-diaminobenzidine (Dako REAL EnVision Detection System, DAKO) was used for visualization and counterstained with Meyer's hematoxylin.

Staining intensities in tumor cells or stroma were independently scored from 0 to 3 (Supplementary Fig.S1) by two different evaluators who were unaware of the clinical findings, and the cases were divided into high (score $=2$ or $>2$ ) and low (score $<2$ ) expression groups by the mean score of the two evaluators. This study protocol was approved by the institutional IRB of Jichi Medical University (Rin A17-164) and conducted in accordance with the guiding principles of the Declaration of Helsinki. Written informed consent was obtained from all participants.

Statistical Analysis

Data are presented as the means \pm SEM or median (min-max). Statistical differences were analyzed by student-t-tests, the Mann-Whitney test, or one-way ANOVA with post hoc test with Tukey's or Dunnett's procedure and $p$ values less than 0.05 were considered significant. Recurrence-free survival rates were calculated using the Kaplan-Meier method and differences were evaluated using the log-rank test.

\section{Results}

RT enhances the expression of CD73 by LuM-1 cells and increases adenosine levels in subcutaneous tumors 
The expression of CD39 and CD73 in cultured LuM-1cells was examined with flow cytometry. CD39 was scarcely expressed on LuM-1 cells and not changed after RT (Fig. 1A, left panel). In comparison, LuM-1 cells positively expressed CD73 and its expression level was further enhanced 24 hours after treatment with 10 Gy RT (Fig. 1A, right panel and Fig.S1-A). RT (4 Gy x2) was given to sc LuM-1 tumors implanted in syngeneic mice, and CD73 expression in the LuM-1 cells recovered from resected tumors were evaluated by mean fluorescein intensity (MFI) in CD45(-) tumor cells. Consistent with the in vitro results, CD73 expression level in LuM-1 cells was significantly enhanced compared with non-irradiated controls (Fig. 1B). MFI in CD45(+) cells did not show significant difference (Fig.S1-B)

The levels of adenosine, as well as its precursor, adenosine monophosphate (AMP) and its metabolite, inosine, in irradiated sc tumors were examined using an LC-MS/MS system. As evaluated by the peak height ratio against the internal standard, adenosine levels in tumors were significantly increased at 24 hours after 2 cycles of RT, and inosine levels were significantly increased at 48 hours after RT (Fig. 1C).

Anti-CD73 mAb combined with RT suppresses non-irradiated lung metastases as well as irradiated tumor

In a preliminary experiment, we confirmed that all mice developed sc tumors with micrometastases in both lungs at 12 days after sc injection of LuM-1 cells, although all mice were healthy with apparent subcutaneous tumor. When local RT (4 Gy x 3) was delivered selectively to sc tumors after 12 days, the weight of the sc tumor at day 28 was significantly reduced $(2.5 \pm 0.61 \mathrm{~g}$ vs $4.8 \pm 0.61 \mathrm{~g}, \mathrm{p}<0.05, \mathrm{n}=5)$, while the number of lung metastases was not altered. Treatment with anti-CD73 mAb alone did not show significant difference from isotype control for the sc tumor or the lung metastases (Fig. 2).

However, when RT was delivered to sc tumor together with administration of anti-CD73 mAb or isotype control, the growth of sc tumor was significantly delayed in mice treated with anti-CD73 mAb $(p<0.05$, at day 18 and later) and tumor volume at day 28 was reduced to about 50\% (Fig. 3A, B). Moreover, the number of lung metastases was significantly reduced in anti-CD73 mAb treated mice (median $=0.5,0 \sim$ 30 vs median $=12,1 \sim 70, p=0.04, n=8)$. No metastases were observed in 4/8 mice treated with RT + anti-CD73 mAb, although metastases developed in all mice in the control group (Fig. 3C, D). Same trend was observed in 2 different experiments with 2 cycles of RT although the differences were not statistically significant (Fig.S2 and Fig.S3).

Anti-CD73 mAb combined with RT enhances the systemic immune response

We then examined lymphocyte populations in the spleens of tumor-bearing mice. The ratios of CD4(+) or CD8(+) T cells and CD11b(+) Gr-1(+) myeloid derived suppressor cells were not altered comparing the anti-CD73 mAb treated and isotype control groups (Fig.S4). However, as shown in Figs. 4A and B, intracellular staining showed that IFN-y producing cells were significantly increased in CD4(+) and CD8(+) T cells in anti-CD73 mAb treated mice (CD4; $10.8 \pm 1.2 \%$ vs $4.7 \pm 1.6 \%, p<0.05, n=6$ : CD8; $16.2 \pm 1.7 \%$ vs $6.9 \pm 2.3 \%, p<0.05, n=6)$. Moreover, infiltrating lymphocytes in sc tumor showed the same trend with statistical significance in CD4(+) population (Fig.S5). When mice were treated in RT or anti-CD73 mAb 
alone in different series of experiment, the ratio of IFN-y producing splenic T cells were much less than those in mice which received the combination of RT and anti-CD73 mAb (Fig.S6).

After co-culture with irradiated LuM-1 cells and rlL-2 for 12 days, splenocytes in RT and anti-CD73 mAb treated mice tended to show increased cytotoxicity against LuM-1 with marginal significance $(12.8 \pm 1.5 \%$ vs $7.8 \pm 2.8 \%$ at $E / T$ ratio $=20, p=0.053, n=3)($ Fig. $4 C$ ).

Expression of CD73 in tumor cells or stroma correlates with the outcomes of patients who received neoadjuvant RT

The expression of CD73 in 64 surgically resected specimens from patients with RC who had received neoadjuvant chemo RT was immunohistochemically evaluated. The outcomes of these patients was evaluated with regard to CD73 expression. As shown in Fig. 5, remnant cancer cells and stroma were stained positive for CD73 and the staining pattern was highly variable among the patients. Therefore, we separately evaluated the staining intensity in remnant tumor cells and stroma (Fig.S7) and divided these into high and low expression groups (Fig. 5 and Table 1). The CD73 expression level did not show significant correlation with clinical or pathological findings including pathological response. However, recurrence in distant sites tended to be observed frequently in patients with higher-expressing CD73 tumors. Accordingly, patients with tumors showing high CD73 expression either in tumor cells or stroma tended to have shorter recurrence-free survival (RFS) and overall survival (OS) compared to patients with low CD73 expression (Fig. 6). Especially, 13 patients with tumors that highly express CD73 both in tumor cells and stroma showed markedly worse outcomes compared to the other 51 patients $(p=0.0059)$ with mean RFS of 22 months (Fig. 6 right panels).

\section{Discussion}

RT has been widely used for the treatment of solid tumors either with curative intent or as palliative treatment. Recent clinical $(13,14)$ as well as pre-clinical $(10-12)$ studies have suggested that tumor responses to RT are significantly enhanced by combination with immune checkpoint blockade.

Adenosine has a strong immunosuppressive property and is now considered as an important "metabolic immune checkpoint molecule" $(22,34)$. Inhibition of the CD73/adenosine axis attracts attention as a novel form of immunotherapy that could be combined with RT $(35,36)$. However, it is unclear how the modulation of adenosine levels affect the outcome of RT.

In this study, we found that CD73 is significantly expressed in a highly metastatic clone of colon26, LuM1 , and was further upregulated by irradiation both in vitro and in vivo. Previous studies have shown that CD73 gene expression is enhanced by hypoxia (37) and proinflammatory cytokines (38) which are often associated with RT. RT has been shown to upregulate CD73 expression in esophageal (39) and bladder cancer (40) cells as well as immune cells (41), which is consistent with the present results. Since after RT large amounts of adenosine precursors are expected to be released into the extracellular space from damaged cells, it is possible that upregulation of CD73 causes large amounts of adenosine to accumulate in irradiated tumor tissue. 
Accurate quantification of tissue adenosine levels is challenging because of its low molecular weight, high polarity and short half-life due to enzymatic degradation (42). Previous studies using reversed phase high pressure liquid chromatography showed that extracellular adenosine levels in solid tumors were 50$100 \propto \mathrm{M}$, which is higher than those in normal tissue and enough to suppress local antitumor immune responses $(43,44)$. In this study, we used the LC-MS method with superior sensitivity and selectivity compared with conventional liquid chromatography (45), and found that adenosine levels in sc LuM-1 tumors are significantly elevated 24 hours after RT. To the best of our knowledge, this is the first report to directly evaluate changes in adenosine levels in irradiated tumors. Levels of inosine, a stable metabolite of adenosine, were increased at a later time. These results suggest that adenosine levels in the microenvironment of irradiated tumors are maintained at considerably high levels, at least for hours, which may attenuate the anti-tumor immune response elicited by RT.

In this study, RT (4 Gy $x$ 3) delayed the growth of sc LuM-1 tumors while anti-CD73 antibody did not show anti-tumor effects when used alone. However, when combined with RT, antibody administration further suppressed the growth of irradiated tumors compared with tumor growth in isotype control treated mice. Anti-CD73 antibody significantly reduced the number of metastases in the lungs, which had not been irradiated. No metastases were observed in the lungs of $50 \%$ of mice treated with anti-CD73 together with RT. Since microscopic metastases already existed in the lungs at the time of treatment, it is suggested that the combination of RT and anti-CD73 antibody evokes a systemic immune response which eliminated tumor cells in the lung. Splenocytes from mice treated with RT and anti-CD73 antibody had an increased ability to produce IFN-y and enhanced cytotoxicity against autologous LuM-1 in vitro. These results suggest that anti-CD73 antibody can induce abscopal effects of RT, which might be partially attributed to T cells stimulated by RT-induced tumor-associated antigen.

CD73 is a multifunctional molecule expressed in various cells. Previous studies have shown that CD73 on tumor cells can mediate proliferation and migration apart from its enzymatic activity and that blocking CD73 can suppress tumor growth $(46,47)$. In other studies, CD73 has been shown to contribute to the process of angiogenesis via both its enzymatic and non-enzymatic functions $(48,49)$. These results suggest that CD73 blockade may suppress the growth of lung metastases through mechanisms unrelated to immunity. In this study, however, it seems to be unlikely because anti-CD73 mAb, when used alone, did not show significant inhibition in lung metastases in vivo. In fact, in vitro proliferation and migration of LuM-1 cells were not affected by CD73 mAb treatment (data not shown).

Immunostaining experiments showed that CD73 was expressed both in remnant tumor cells and/or stroma in surgically resected human RC after CRT. Although the expression pattern differs among patients, high expression of CD73 was associated with poor prognosis with a higher incidence of distant recurrence, which is consistent with previous studies of non-irradiated tumors $(23)(24,25)$. This might be partially caused by the concurrent chemotherapy, since chemotherapy induced CD73 expression in epithelial ovarian cancer and CD73 blockade improved the therapeutic efficacy (50). However, together with the results of the murine experiments, it is suggested that increased adenosine levels, by enhanced 
CD73 in irradiated tumor tissue, may impair systemic immune responses which might be causally related to the growth of micrometastases in distant organs in human.

There is growing evidence that RT can result in in situ tumor vaccination by exposing tumor specific neoantigens to the host innate immune system, and thus radio- immunotherapy has the possibility of being an effective novel therapy for patients with advanced cancer. However, there are still major challenges to understanding the dual face of RT-induced effects on the immune system. This is the first report to suggest that the anti-tumor response may be reduced by adenosine in irradiated tumor which is restored by functional blockade of $\mathrm{CD} 73$. Anti-CD73 mAb has already been used in a phase 1 clinical trial (NCT02503774) (51). These results of the present study encourage the clinical appreciation of anti-CD73 $\mathrm{mAb}$ combined with RT as a promising preoperative treatment for patients with locally advanced RC.

\section{Conclusion}

After local RT, adenosine levels in irradiated tumor is considerably elevated which may reduce the antitumor effects mediated by RT through the induction of immunosuppression. The combination with CD73/adenosine axis blockade may enhance local and abscopal effects of RT and improve the outcomes of patients with locally advanced rectal cancer.

\section{Abbreviations}

RT: radiation, ip: intraperitoneal,

\section{Declarations}

\section{Ethics approval and consent to participate}

Animal experiment procedures were approved by Animal Care Committee of Jichi Medical University (No 17005-02) and performed according to the Japanese Guidelines for Animal Research.

Immunohistochemistry study protocol was approved by the institutional IRB of Jichi Medical University (Rin A17-164) and written informed consent to participate in this study was obtained from all participants.

\section{Consent for publication}

Written informed consent for publication was also obtained from all participants.

\section{Availability of data and materials}

'Not applicable'

Competing interests 
Yasunaru Sakuma is an Associate Editor and Alan Kawarai Lefor is a Section Editor.

\section{Funding}

This study was supported by Japan Society for the Promotion of Science (17K10649, 19K09225) in animal experiments and adenosine quantification. This study was also supported by Keirin Race Fund from JKA foundation in flowcytometric analysis using LSRFortessa.

\section{Authors' contributions}

$\mathrm{HT}, \mathrm{H} . \mathrm{H}, \mathrm{K} . \mathrm{K}$ and J.K. conceived and designed the experiments. $\mathrm{HT}, \mathrm{HO}$ and $\mathrm{YH}$ performed animal experiments. HT. YS, H.H, K.K and HY provided the clinical samples. KY provided the experimental system. AKL, NS and J.K wrote the main manuscript. All authors have read and approved the manuscript.

\section{Acknowledgements}

We appreciate Dr K. Oguri for providing LuM-1 and Dr. Prof. T. Niki for his proper advice about the evaluation of immunohistochemistry. We also thank Ms. H. Hayakawa, J. Shinohara, H. Hatakeyama, N. Nishiaki and I. Nieda for technical and clerical works.

\section{References}

1. Kapiteijn E, Marijnen CA, Nagtegaal ID, Putter H, Steup WH, Wiggers T, et al. Preoperative radiotherapy combined with total mesorectal excision for resectable rectal cancer. N Engl J Med. 2001;345(9):63846.

2. Erlandsson J, Martling A. Short-course radiotherapy with delayed surgery for rectal cancer - Authors' reply. Lancet Oncol. 2017;18(6):e295.

3. Bosset JF, Collette L, Calais G, Mineur L, Maingon P, Radosevic-Jelic L, et al. Chemotherapy with preoperative radiotherapy in rectal cancer. N Engl J Med. 2006;355(11):1114-23.

4. O'Connell MJ, Colangelo LH, Beart RW, Petrelli NJ, Allegra CJ, Sharif S, et al. Capecitabine and oxaliplatin in the preoperative multimodality treatment of rectal cancer: surgical end points from National Surgical Adjuvant Breast and Bowel Project trial R-04. Journal of clinical oncology : official journal of the American Society of Clinical Oncology. 2014;32(18):1927-34.

5. Barcellos-Hoff MH, Park C, Wright EG. Radiation and the microenvironment - tumorigenesis and therapy. Nat Rev Cancer. 2005;5(11):867-75.

6. Clifford R, Govindarajah N, Parsons JL, Gollins S, West NP, Vimalachandran D. Systematic review of treatment intensification using novel agents for chemoradiotherapy in rectal cancer. The British journal of surgery. 2018;105(12):1553-72.

7. Gulley JL, Arlen PM, Bastian A, Morin S, Marte J, Beetham P, et al. Combining a recombinant cancer vaccine with standard definitive radiotherapy in patients with localized prostate cancer. Clin Cancer 
Res. 2005;11(9):3353-62.

8. Golden EB, Frances D, Pellicciotta I, Demaria S, Helen Barcellos-Hoff M, Formenti SC. Radiation fosters dose-dependent and chemotherapy-induced immunogenic cell death. Oncoimmunology. 2014;3:e28518.

9. Formenti SC, Demaria S. Systemic effects of local radiotherapy. Lancet Oncol. 2009;10(7):718-26.

10. Park SS, Dong H, Liu X, Harrington SM, Krco CJ, Grams MP, et al. PD-1 Restrains RadiotherapyInduced Abscopal Effect. Cancer Immunol Res. 2015;3(6):610-9.

11. Demaria S, Golden EB, Formenti SC. Role of Local Radiation Therapy in Cancer Immunotherapy. JAMA Oncol. 2015;1(9):1325-32.

12. Dovedi SJ, Adlard AL, Lipowska-Bhalla G, McKenna C, Jones S, Cheadle EJ, et al. Acquired resistance to fractionated radiotherapy can be overcome by concurrent PD-L1 blockade. Cancer Res. 2014;74(19):5458-68.

13. Antonia SJ, Villegas A, Daniel D, Vicente D, Murakami S, Hui R, et al. Durvalumab after Chemoradiotherapy in Stage III Non-Small-Cell Lung Cancer. N Engl J Med. 2017;377(20):1919-29.

14. Shaverdian N, Lisberg AE, Bornazyan K, Veruttipong D, Goldman JW, Formenti SC, et al. Previous radiotherapy and the clinical activity and toxicity of pembrolizumab in the treatment of non-smallcell lung cancer: a secondary analysis of the KEYNOTE-001 phase 1 trial. Lancet Oncol. 2017;18(7):895-903.

15. Kwon ED, Drake CG, Scher HI, Fizazi K, Bossi A, van den Eertwegh AJ, et al. Ipilimumab versus placebo after radiotherapy in patients with metastatic castration-resistant prostate cancer that had progressed after docetaxel chemotherapy (CA184-043): a multicentre, randomised, double-blind, phase 3 trial. Lancet Oncol. 2014;15(7):700-12.

16. Luke JJ, Lemons JM, Karrison TG, Pitroda SP, Melotek JM, Zha Y, et al. Safety and Clinical Activity of Pembrolizumab and Multisite Stereotactic Body Radiotherapy in Patients With Advanced Solid Tumors. Journal of clinical oncology : official journal of the American Society of Clinical Oncology. 2018;36(16):1611-8.

17. Hasko G, Cronstein BN. Adenosine: an endogenous regulator of innate immunity. Trends Immunol. 2004;25(1):33-9.

18. Antonioli L, Blandizzi C, Pacher P, Hasko G. Immunity, inflammation and cancer: a leading role for adenosine. Nat Rev Cancer. 2013;13(12):842-57.

19. Sorrentino R, Pinto A, Morello S. The adenosinergic system in cancer: Key therapeutic target. Oncoimmunology. 2013;2(1):e22448.

20. Di Virgilio F, Adinolfi E. Extracellular purines, purinergic receptors and tumor growth. Oncogene. 2017;36(3):293-303.

21. Kaufmann A, Musset B, Limberg SH, Renigunta V, Sus R, Dalpke AH, et al. "Host tissue damage" signal ATP promotes non-directional migration and negatively regulates toll-like receptor signaling in human monocytes. J Biol Chem. 2005;280(37):32459-67. 
22. Ohta A. A Metabolic Immune Checkpoint: Adenosine in Tumor Microenvironment. Front Immunol. 2016;7:109.

23. Wu XR, He XS, Chen YF, Yuan RX, Zeng Y, Lian L, et al. High expression of CD73 as a poor prognostic biomarker in human colorectal cancer. Journal of surgical oncology. 2012;106(2):130-7.

24. Ren ZH, Yuan YX, Ji T, Zhang CP. CD73 as a novel marker for poor prognosis of oral squamous cell carcinoma. Oncol Lett. 2016;12(1):556-62.

25. Inoue $Y$, Yoshimura $K$, Kurabe $N$, Kahyo $T$, Kawase $A$, Tanahashi $M$, et al. Prognostic impact of CD73 and A2A adenosine receptor expression in non-small-cell lung cancer. Oncotarget. 2017;8(5):8738-51.

26. Allard B, Pommey S, Smyth MJ, Stagg J. Targeting CD73 enhances the antitumor activity of anti-PD1 and anti-CTLA-4 mAbs. Clin Cancer Res. 2013;19(20):5626-35.

27. Beavis PA, Divisekera U, Paget C, Chow MT, John LB, Devaud C, et al. Blockade of A2A receptors potently suppresses the metastasis of CD73+tumors. Proc Natl Acad Sci U S A. 2013;110(36):14711-6.

28. Young A, Ngiow SF, Barkauskas DS, Sult E, Hay C, Blake SJ, et al. Co-inhibition of CD73 and A2AR Adenosine Signaling Improves Anti-tumor Immune Responses. Cancer Cell. 2016;30(3):391-403.

29. Antonioli L, Yegutkin GG, Pacher P, Blandizzi C, Hasko G. Anti-CD73 in cancer immunotherapy: awakening new opportunities. Trends Cancer. 2016;2(2):95-109.

30. Leone RD, Emens LA. Targeting adenosine for cancer immunotherapy. J Immunother Cancer. 2018;6(1):57.

31. Sakata K, Kozaki K, lida K, Tanaka R, Yamagata S, Utsumi KR, et al. Establishment and characterization of high- and low-lung-metastatic cell lines derived from murine colon adenocarcinoma 26 tumor line. Jpn J Cancer Res. 1996;87(1):78-85.

32. Yasuda K, Nirei T, Tsuno NH, Nagawa H, Kitayama J. Intratumoral injection of interleukin-2 augments the local and abscopal effects of radiotherapy in murine rectal cancer. Cancer science. 2011;102(7):1257-63.

33. Jimmerson LC, Bushman LR, Ray ML, Anderson PL, Kiser JJ. A LC-MS/MS Method for Quantifying Adenosine, Guanosine and Inosine Nucleotides in Human Cells. Pharm Res. 2017;34(1):73-83.

34. Vaupel P, Multhoff G. Commentary: A Metabolic Immune Checkpoint: Adenosine in Tumor Microenvironment. Front Immunol. 2016;7:332.

35. Vaupel P, Multhoff G. Adenosine can thwart antitumor immune responses elicited by radiotherapy : Therapeutic strategies alleviating protumor ADO activities. Strahlenther Onkol. 2016;192(5):279-87.

36. de Leve S, Wirsdorfer F, Jendrossek V. Targeting the Immunomodulatory CD73/Adenosine System to Improve the Therapeutic Gain of Radiotherapy. Front Immunol. 2019;10:698.

37. Synnestvedt K, Furuta GT, Comerford KM, Louis N, Karhausen J, Eltzschig HK, et al. Ecto-5'nucleotidase (CD73) regulation by hypoxia-inducible factor- 1 mediates permeability changes in intestinal epithelia. J Clin Invest. 2002;110(7):993-1002.

38. Gao ZW, Dong K, Zhang HZ. The roles of CD73 in cancer. Biomed Res Int. 2014;2014:460654. 
39. Fukuda K, Sakakura C, Miyagawa K, Kuriu Y, Kin S, Nakase Y, et al. Differential gene expression profiles of radioresistant oesophageal cancer cell lines established by continuous fractionated irradiation. British journal of cancer. 2004;91(8):1543-50.

40. Dietrich F, Figueiro F, Filippi-Chiela EC, Cappellari AR, Rockenbach L, Tremblay A, et al. Ecto-5'nucleotidase/CD73 contributes to the radiosensitivity of T24 human bladder cancer cell line. J Cancer Res Clin Oncol. 2018;144(3):469-82.

41. Mandapathil M, Szczepanski MJ, Szajnik M, Ren J, Lenzner DE, Jackson EK, et al. Increased ectonucleotidase expression and activity in regulatory T cells of patients with head and neck cancer. Clin Cancer Res. 2009;15(20):6348-57.

42. Goodwin KJ, Gangl E, Sarkar U, Pop-Damkov P, Jones N, Borodovsky A, et al. Development of a quantification method for adenosine in tumors by LC-MS/MS with dansyl chloride derivatization. Anal Biochem. 2019;568:78-88.

43. Blay J, White TD, Hoskin DW. The extracellular fluid of solid carcinomas contains immunosuppressive concentrations of adenosine. Cancer Res. 1997;57(13):2602-5.

44. Vaupel P, Mayer A. Hypoxia-Driven Adenosine Accumulation: A Crucial Microenvironmental Factor Promoting Tumor Progression. Adv Exp Med Biol. 2016;876:177-83.

45. Sharma K, Singh R, Giri S, Rajagopal S, Mullangi R. Highly sensitive method for the determination of adenosine by LC-MS/MS-ESI: method validation and scope of application to a pharmacokinetic/pharmacodynamic study. Biomed Chromatogr. 2012;26(1):81-8.

46. Sadej R, Skladanowski AC. Dual, enzymatic and non-enzymatic, function of ecto-5'-nucleotidase (eN, CD73) in migration and invasion of A375 melanoma cells. Acta Biochim Pol. 2012;59(4):647-52.

47. Gao ZW, Wang HP, Lin F, Wang X, Long M, Zhang HZ, et al. CD73 promotes proliferation and migration of human cervical cancer cells independent of its enzyme activity. BMC cancer. 2017;17(1):135.

48. Allard B, Turcotte M, Spring K, Pommey S, Royal I, Stagg J. Anti-CD73 therapy impairs tumor angiogenesis. Int J Cancer. 2014;134(6):1466-73.

49. Ghalamfarsa G, Rastegari A, Atyabi F, Hassannia H, Hojjat-Farsangi M, Ghanbari A, et al. Antiangiogenic effects of CD73-specific siRNA-loaded nanoparticles in breast cancer-bearing mice. J Cell Physiol. 2018;233(10):7165-77.

50. Li H, Lv M, Qiao B, Li X. Blockade pf CD73/adenosine axis improves the therapeutic efficacy of docetaxel in epithelial ovarian cancer. Arch Gynecol Obstet. 2019;299(6):1737-46.

51. Hay CM, Sult E, Huang Q, Mulgrew K, Fuhrmann SR, McGlinchey KA, et al. Targeting CD73 in the tumor microenvironment with MEDI9447. Oncoimmunology. 2016;5(8):e1208875.

\section{Table}


Table 1. CD73 expression levels and clinical and pathological findings of 64 patients with rectal cancer treated with neoadjuvant radiation therapy

Variable

Tumor cells (64)

Stroma (64)

Low (38) High

(22)

Unknown

(4)

value

Low (27)

High

value

Age

63)(36-

$59(42-\quad 66(63-68)$

78)

79)

0.13

61)(36-

$62(44-$

0.8

Gender

$\begin{array}{llllllll}\text { M } & 29 & 16 & 3 & 0.77 & 19 & 29 & 0.56 \\ \text { F } & 9 & 6 & 1 & & 8 & 8 & \end{array}$

Location

Rab

2

1

0.69

2

1

0.48

$\mathrm{Rb}$

36

21

25

36

Histology

Differentiated

32

20

0.7

22

31

0.46

Undifferentiated 6

2

2

6

Lymphatic invasion

\begin{tabular}{lllllll} 
Absent & 24 & 11 & 0.42 & 17 & 22 & 0.8 \\
\hline Present & 14 & 11 & & 10 & 15 &
\end{tabular}

Venous invasion

$\begin{array}{lllllll}\text { Absent } & 13 & 9 & 0.78 & 10 & 16 & 0.8 \\ \text { Present } & 25 & 13 & & 17 & 21 & \end{array}$

Tumor Stage

\begin{tabular}{|llllllll}
\hline t0/t1 & 4 & 0 & 4 & 0.48 & 5 & 3 & 0.35 \\
\hline t2 & 9 & 5 & & 7 & 7 & \\
\hline t3 & 23 & 14 & & 12 & 25 & \\
\hline t4 & 2 & 3 & 3 & 2 & 0.79 \\
\hline N stage & & & & & & 25 & \\
\hline n0 & 23 & 15 & 0.59 & 17 & 10 & 12 & \\
\hline n1< & 15 & 7 & & 10 & & &
\end{tabular}

Pathological response 


\begin{tabular}{|c|c|c|c|c|c|c|c|}
\hline grade $0 / 1$ & 25 & 17 & & 0.29 & 18 & 24 & 0.63 \\
\hline grade 2 & 13 & 5 & 1 & & 7 & 12 & \\
\hline grade 3 & 0 & 0 & 3 & & 2 & 1 & \\
\hline \multicolumn{8}{|c|}{ Combined Chemotherapy } \\
\hline Not given & 3 & 4 & 0 & 0.31 & 4 & 3 & 0.2 \\
\hline Given & 35 & 18 & 4 & & 23 & 34 & \\
\hline \multicolumn{8}{|c|}{ Adjuvant chemotherapy } \\
\hline Given & 16 & 5 & & 0.13 & 9 & 12 & 0.99 \\
\hline Not Given & 22 & 17 & 4 & & 18 & 25 & \\
\hline \multicolumn{8}{|c|}{ Recurrence } \\
\hline Present & 11 & 11 & & 0.16 & 6 & 16 & 0.11 \\
\hline Absent & 27 & 11 & 4 & & 21 & 21 & \\
\hline \multicolumn{8}{|c|}{$\begin{array}{l}\text { CD73 expression in tumor cells cannot be appropriately evaluated in } 1 \text { patient with a grade } 2 \\
\text { response due to few remaining tumor cells as well as in } 3 \text { patients with grade } 3 \text { responses } \\
\text { (pathological complete response). Statistical significance of the differences was evaluated by the } c^{2}- \\
\text { test, Fisher's exact test }\end{array}$} \\
\hline
\end{tabular}

\section{Figures}


A

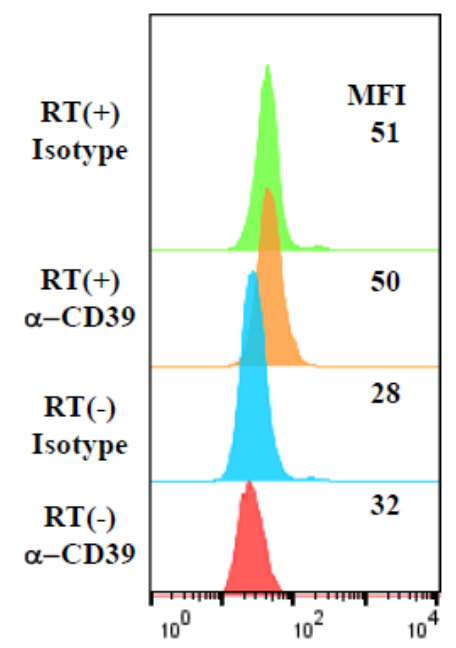

C

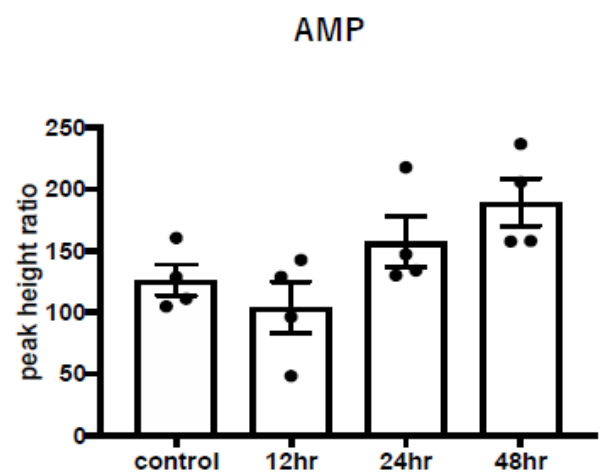

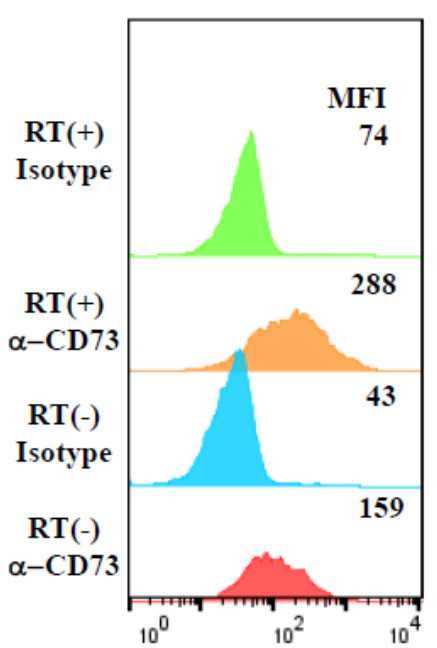

B

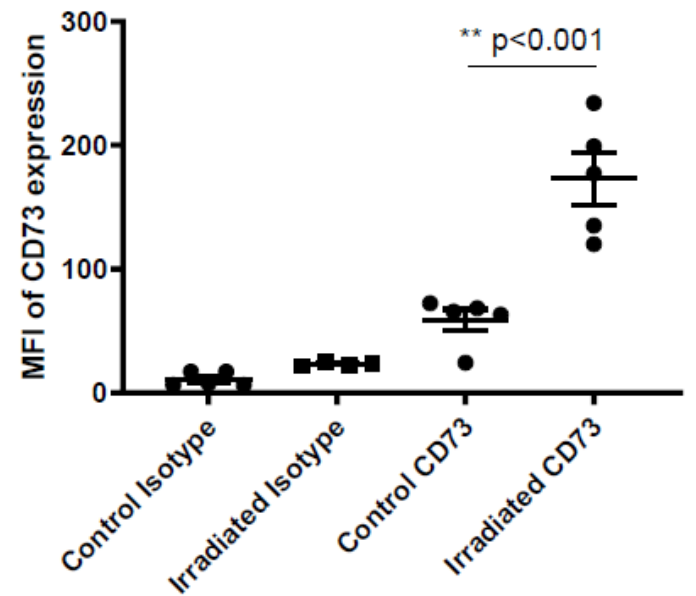

Adenosine

* $\mathrm{p}=0.0069$
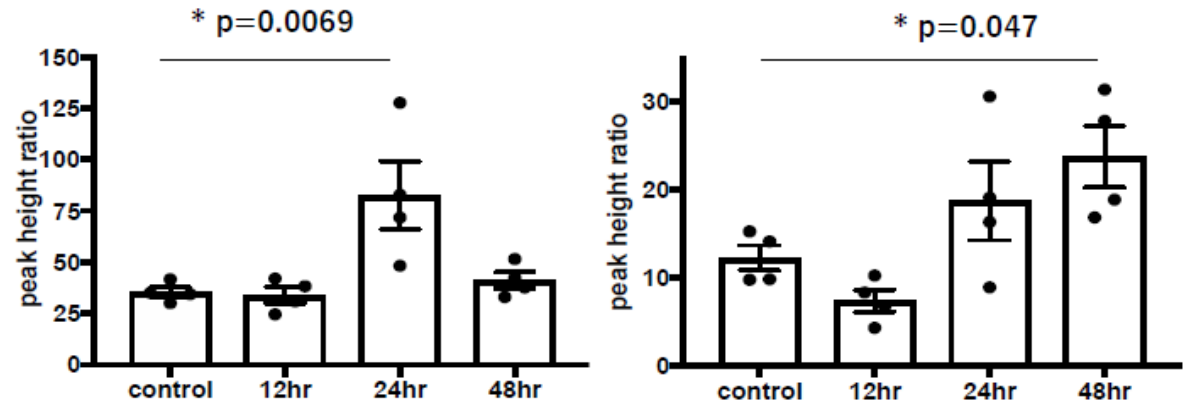

Figure 1

Radiation therapy (RT) enhances the membrane expression of CD73 and increases adenosine levels in subcutaneous (sc) LuM-1 tumors. (A) Cultured LuM-1 cells were treated with or without 10 Gy RT using the MX-160 Labo (mediXtec), and incubated for an additional 24 hours. The cells were stained with mAbs to CD39 (left) or CD73 (right) and mean fluorescein intensities (MFI) in the 7AAD (-) live cell population were examined by FACS. Data show a representative FACS profile in 5 different experiments. (B) Two fractions of 4 Gy RT were delivered selectively to sc tumors of LuM-1 with the remainder of Balb/c mice shielded by a lead plate. Two days later, tumors were resected and single cell suspensions obtained using a Tumor Dissociation Kit (Miltenyi Biotec). The cells were stained with mAbs to CD73 and CD45, and MFI for CD73 were analyzed in live tumor cells defined by 7AAD (-) CD45(-) gated area. P value was calculated with one-way ANOVA followed by Tukey test. (C) Two fractions of 4 Gy RT were delivered to sc tumors as described above, which were removed at 12, 24 and 48 hours after RT. Levels of AMP, adenosine, and inosine levels in those samples were measured with the LC-MS system (Shimadzu Corp, Kyoto, Japan) as described in material and methods. The $Y$ axis shows the height ratio between the 2-morpholinoethanesulfonic acid (2-ME) as an internal standard and the target molecules. $P$ value was calculated with one-way ANOVA followed by Dunnett's test. * $\mathrm{p}<0.05$ 

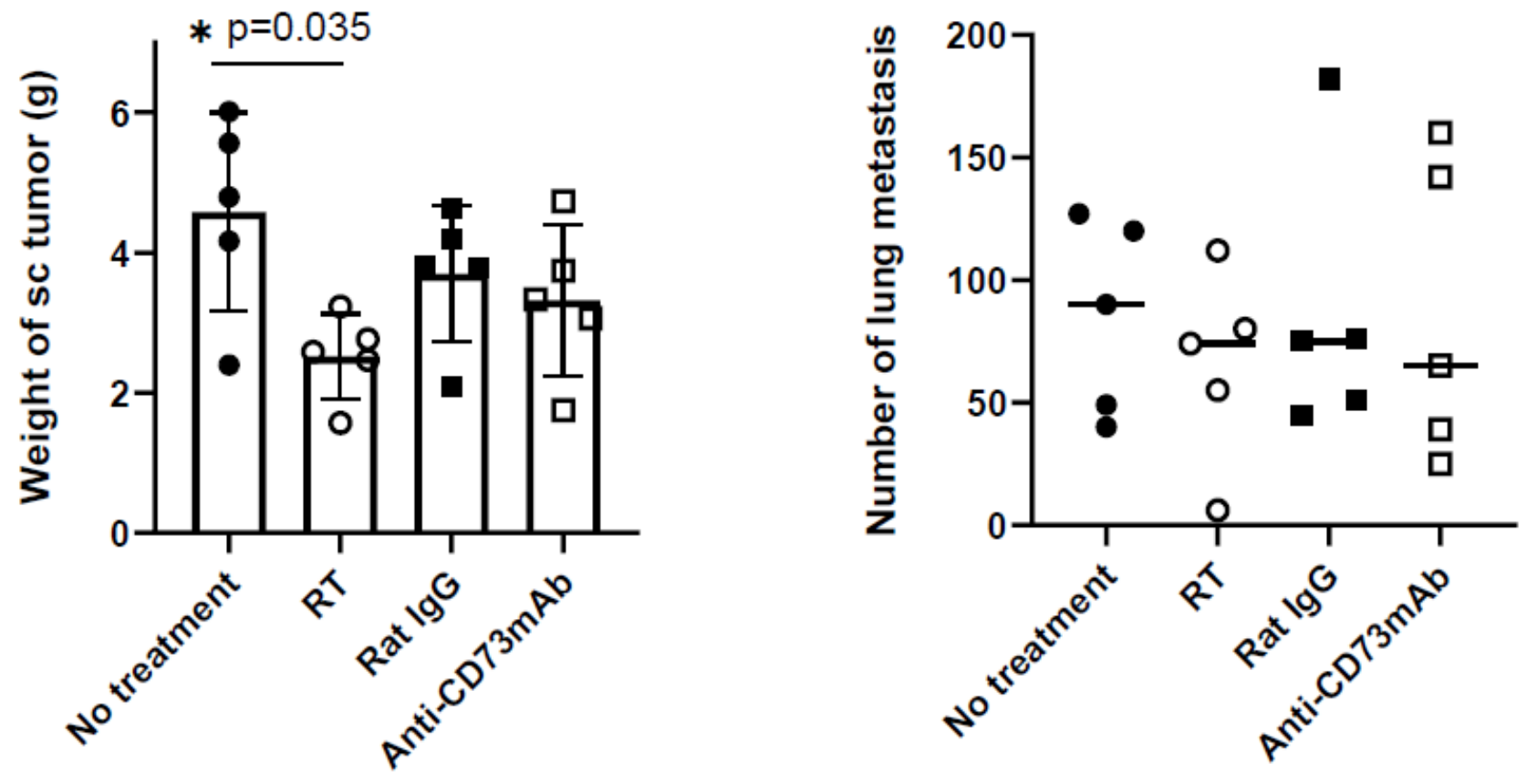

\section{Figure 2}

Anti-tumor effects of radiation therapy (RT) or anti-CD73 antibody used alone. Tumor bearing mice received local RT to subcutaneous (sc) tumors ( 3 fractions of $4 \mathrm{~Gy}$ ) as described above on days 12,14 , 16 and intraperitoneal injection of $200 \mu \mathrm{g}$ anti-CD73 mAb or Rat IgG2a isotype control at days 12, 14, 16, 19,22 and 25 . All of the mice were sacrificed on day 28 , and the weight of sc tumors and number of macroscopic metastatic nodules in the lungs evaluated. : ${ }^{*}<0.05$ 

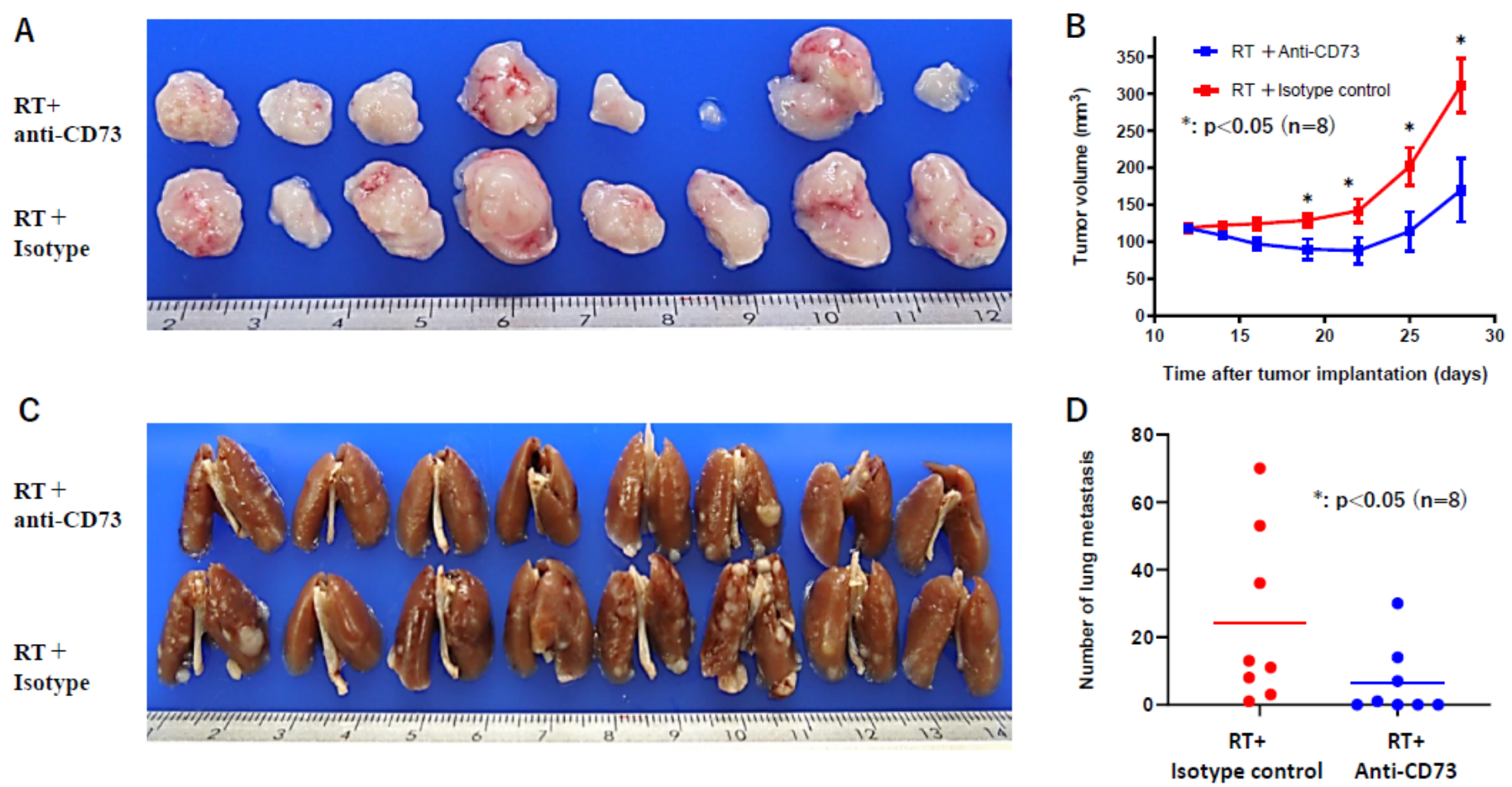

\section{Figure 3}

Anti-tumor effects of anti-CD73 antibody combined with radiation therapy. Tumor bearing mice received local radiation therapy together with immunotherapy using the same protocol shown in Figure 2. The growth of subcutaneous tumors was evaluated by their volume calculated by length $\times$ width $2 / 2$ (B). All mice were sacrificed on day 28 , and the volume of subcutaneous tumor $(A)$ as well as the number of macroscopic metastatic nodules (C, D) in the lungs counted. : ${ }^{*}<0.05$

A

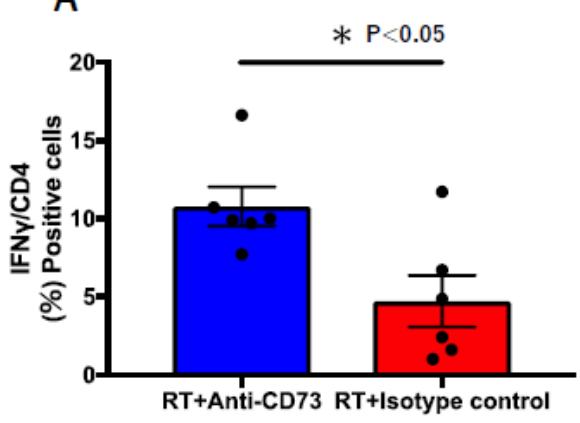

B

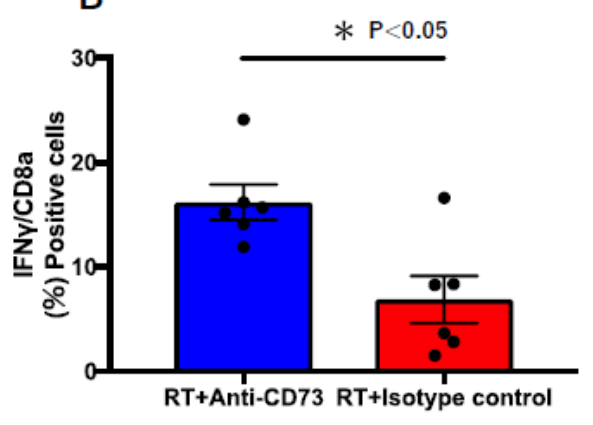

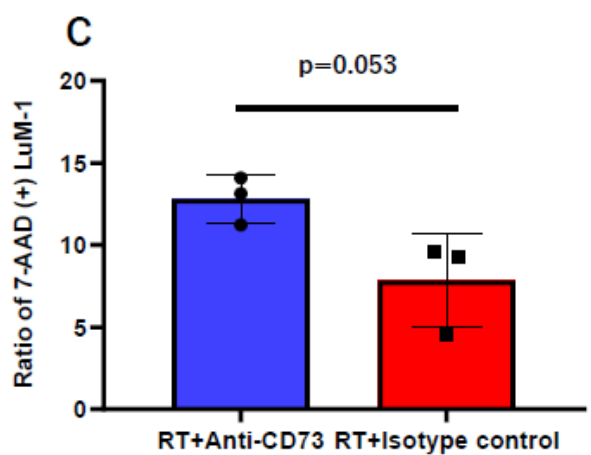

\section{Figure 4}

Effects of anti-CD73 antibody on splenocytes of irradiated tumor bearing mice. CD73 mAb enhances IFN$\triangle$ production (A, B) and cytotoxicity (C) of splenocytes from irradiated mice. (A, B) Tumor bearing mice received 3 fractions of $4 \mathrm{~Gy}$ local radiation therapy together with intraperitoneal injection of $200 \mu \mathrm{g}$ antiCD73 mAb or Rat IgG2a isotype control on days $12,14,16$, and sacrificed at day 18 . The splenocytes 
were cultured in RPMI-1640 + 10\% FCS in the presence of brefeldin and then fixed, permeabilized and stained with PE-conjugated IFN- $\$ or isotype control and FITC-conjugated mAbs to CD4 or CD8 as well as FVS780 for dead cell exclusion. The ratio of IFN-囚 positive cells were calculated in CD4(+) or CD8(+) gated area. (C) The splenocytes were cultured with irradiated LuM1 in 2 ml 10\% FCS+ PRMA-1640 medium supplemented with $20 \mathrm{ng} / \mathrm{ml}$ mouse recombinant IL-2 for 12 days, and then incubated with LuM1 cells at an E/T ratio of 20:1. After 4-hours incubation, all cells were stained with FITC-conjugated Annexin-V, 7-AAD and anti-CD45-APC $m A b$, and ratios of 7-AAD positive dead cells calculated in LuM-1 population defined in FSC/SCC and CD45(-) gated area. *: $p<0.05$

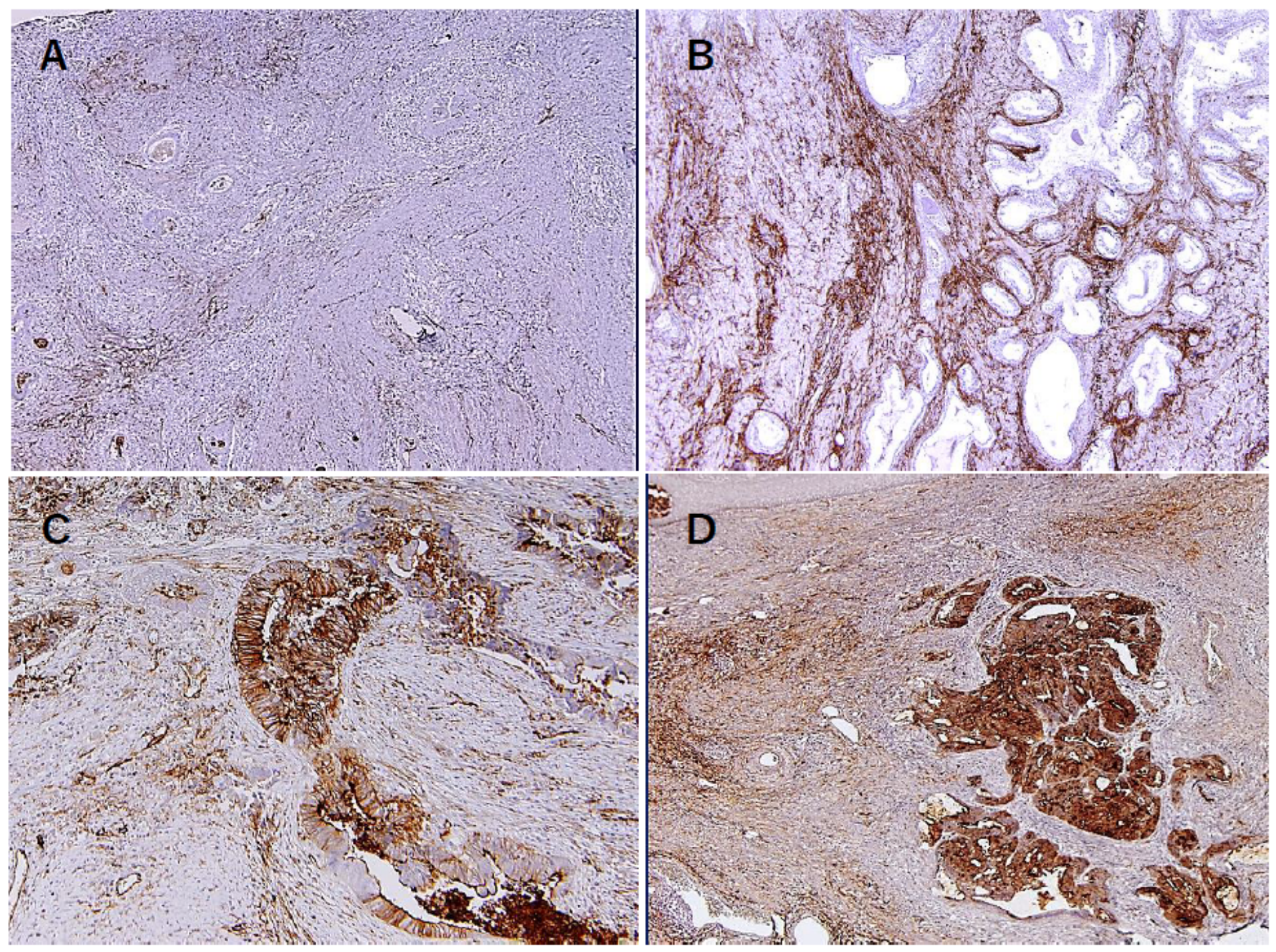

\section{Figure 5}

CD73 expression in rectal cancer tissue after chemoradiation therapy. Formalin-fixed paraffin-embedded 4- $\mu \mathrm{m}$ sections were immune stained with polyclonal Ab to human CD73 using REAL EnVision Detection System (DAKO) as described in Methods. The staining intensities were separately evaluated in tumor cells or stroma and divided into high and low expression groups. Four representative cases, (A) tumor cell low, stroma low (B) tumor cell low, stroma high (C) tumor cell high, stroma low (D) tumor cell high, stroma high were shown. 

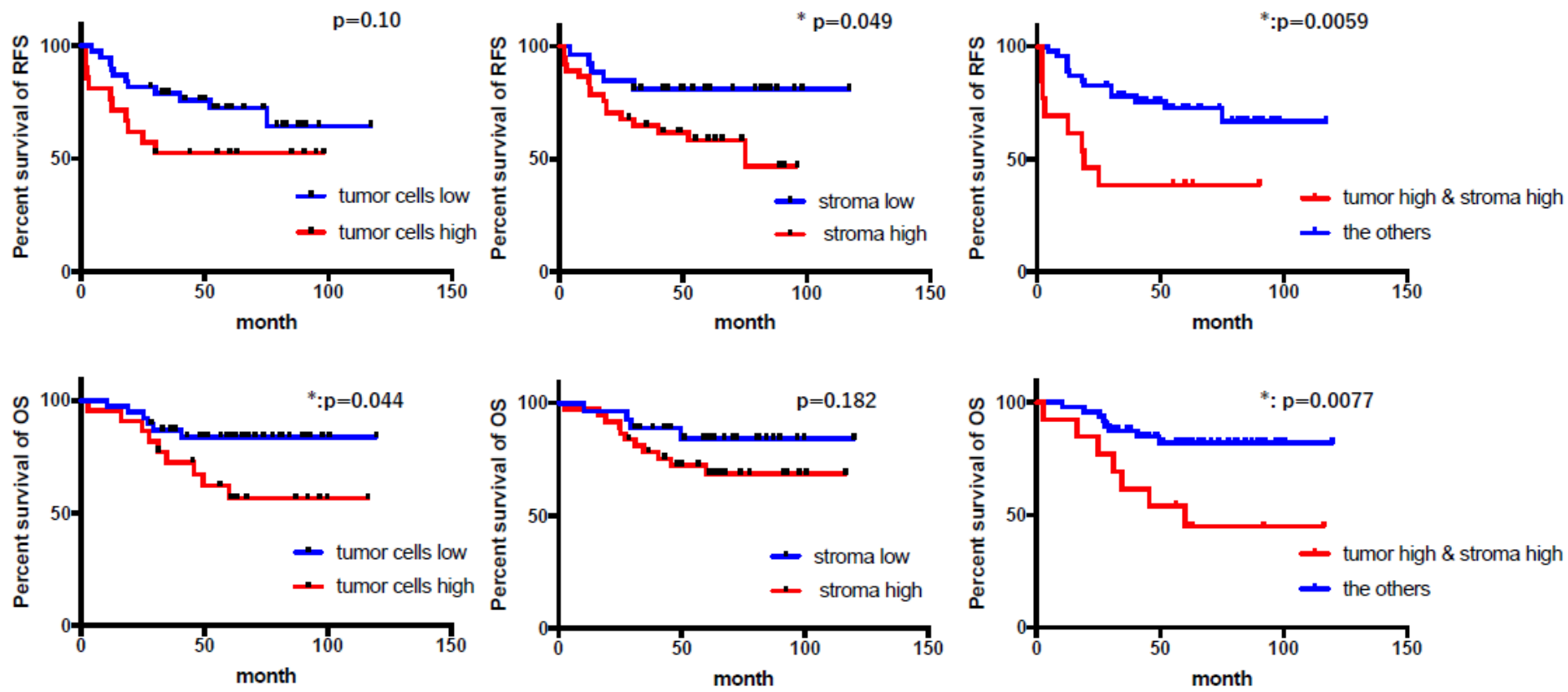

Figure 6

Impact of CD73 expression on outcome of 64 patients who received preoperative RT for locally advanced rectal cancer. Patients were divided into CD73 high and low expression groups either in tumor cells (left panels) or stroma (middle panels), as well as high in both areas or others (right panels), and recurrence free survival (RFS; upper panels) and overall survival (OS; lower panels) were compared with KaplanMeier method. $P$ values were calculated by the log-rank test.

\section{Supplementary Files}

This is a list of supplementary files associated with this preprint. Click to download.

- NC3RsARRIVEGuidelinesChecklistfillable.pdf

- Supplementarydata.revpptx.pdf 